\title{
Knowledge, attitudes and practices about rabies prevention and control: A community survey in five health districts of Burkina Faso
}

\author{
Madi SAVADOGO ${ }^{1,5,6^{*}}$, Estelle KANYALA ${ }^{3}$, Adèle KAM TRAORÉ3 \\ Laibané Dieudonné DAHOUROU ${ }^{2}$, Hyacinthe Victor GUIGMA ${ }^{4}$, \\ Sandaogo Hamidou OUANDAOGO ${ }^{4}$, Abdoul Salam OUEDRAOGO ${ }^{4}$, \\ Grégorie Hermann BAZIMO ${ }^{4}$, Joseph SAVADOGO ${ }^{4}$, Rianatou BADA ALAMBEDJI ${ }^{5}$ and \\ Zékiba TARNAGDA ${ }^{1}$
}

${ }^{1}$ Unité des Maladies à potentiel Epidémique, Maladies Emergentes et Zoonoses, Département de Biologie Médicale et Santé Publique, Institut de Recherche en Sciences de la Santé (IRSS/CNRST), 01 BP 545, Bobo Dioulaso, Burkina Faso.

${ }^{2}$ Institut Supérieur de l'Environnement et du Développement Durable, Université de Dédougou (UDDG), 01 BP 176, Dédougou, Burkina Faso.

${ }^{3}$ Centre d'Urgence pour la lutte contre les Maladies Animales Transfrontières, Organisation des Nations Unies pour l'Agriculture et l'Alimentation (FAO), 01 BP 2540, Ouagadougou, Burkina Faso.

${ }^{4}$ Direction Générale des Services Vétérinaires, Ministère des Ressources Animales et Halieutiques (MRAH), 01 BP 7068, Ouagadougou, Burkina Faso.

${ }^{5}$ Service de Microbiologie Immunologie et Pathologie Infectieuse, Département de Santé Publique et Environnement, Ecole Inter-Etats des Sciences et Médecine Vétérinaires (EISMV), BP 5077, Senegal

${ }^{6}$ Fundamental and Applied Research for Animals and Health (FARAH), Faculty of Veterinary Medicine, University of Liege, 4000, Liege, Belgium.

*Corresponding author ; E-mail : savadogo.madi@yahoo.fr; 01 BP 545, Bobo Dioulasso, Burkina Faso.

Received: 08-04-2021

Accepted: 27-09-2021
Published: $30-10-2021$

\begin{abstract}
Rabies is a deadly viral disease that affects humans and animals. It is transmitted by rabid animals, through bite, scratch or licking. Almost $99 \%$ of human cases are caused by dogs. In Burkina Faso, nearly 5,000 bites cases are recorded annually. This cross-sectional investigation was conducted with the objective of assessing the knowledge, attitudes and practices of people regarding rabies and preventive measures in five health districts, Burkina Faso. Using a structured questionnaire, the survey was conducted in households to capture data on participants' knowledge, attitudes and practices of rabies control. Through a house-to-house approach, the questionnaire was administrated to 320 household members. The results indicated that $36.9 \%$ of the participants had high level knowledge of rabies, $80 \%$ perceived it as a fatal disease, $76.4 \%$ of participant's reported attitudes were negative and $58.9 \%$ of them reported uncorrect practices. The knowledge level of rabies was significantly associated with health district, area of household location, participant's age, their level of education, previous
\end{abstract}


history with rabies and information channels used by household $(\mathrm{p}<0.05)$. However, the health district, the area of household location, the level of education and the knowledge level had significant association with attitudes of participants with regard to rabies $(\mathrm{p}<0.05)$. Concerning health behaviours, health district of respondents and their previous history with rabies showed significant association with practices regarding bite cases management $(\mathrm{p}<0.05)$. In addition, people health seeking behaviors were significantly associated with their levels of knowledge regarding rabies, their perceptions and attitudes towards the disease $(\mathrm{p}<0.05)$. National rabies control stakeholders should address the knowledge gaps through mass awareness campaigns providing key information regarding the risks of contamination and the recommended practices to prevent avoidable deaths.

(C) 2021 International Formulae Group. All rights reserved.

Keywords: Burkina Faso, household survey, rabies control, knowledge of rabies, community behaviours.

\section{INTRODUCTION}

Rabies is a transmissible infectious zoonosis, caused by a neurotropic Lyssavirus (Sabeta et al., 2003). It is a fatal and irreversible disease once the clinical signs appear. Rabies affects humans as well as many species of domestic and wild mamals. The transmission to human or animal occurs through a close contact (bite, scraching, licking) with the saliva of a rabid animal. It is a high socio-economic impact zoonotic disease, mostly in Africa and Asia (Hampson et al., 2015). Indeed, rabies related mortality is globally estimated at about 59,000 human deaths each year and economic impact of the disease at nearly USD 8,6 billion (Hampson et al., 2015). About $45 \%$ of human deaths occur in Africa (Dodet et al., 2010), with almost 2,000 deaths in North Africa, 7,000 in Central Africa, 6,000 in Southern Africa and 6,000 in West Africa (Hampson et al., 2015).

In Burkina Faso, despite known underreporting of rabies, an average of 5,000 bites and 5 deaths is annually recorded (Sondo et al., 2015, Savadogo et al., 2020). Being a zoonosis, rabies surveillance and control involves a diversity of stakeholders according to the One Health approach's principles. Therefore, national surveillance and control strategy should foster collaboration between sectors including human health, animal health and wildlife management sectors (Zangre et al., 2018 ; Acharya et al., 2020). In 2017, during zoonotic diseases prioritization (ZDP) process, these key sectors jointly identified rabies as one of the top 5 priority zoonosis countrywide. Rabies has been a mandatory notifiable disease in Burkina Faso for decades (Burkina Faso, 2018). The control of the disease is based on domestic pets vaccination and human postexposure prophylaxis. The National Livestock Laboratory (Laboratoire National d'Elevage, located in Ouagadougou) is in charge of testing samples collected from suspected rabid animals (Sondo et al., 2014 ; Savadogo et al., 2020; Minoungou et al., 2021), while private and public veterinarians provide domestic pets rabies vaccination. Some veterinarians holding mandate from the animal health authorities, are in charge of sanitary observation of biting pets as required in bite cases management and postexposure prophyxis (PEP). The PEP is provided by two Rabies Treatment Centers (Centre de Traitement Antirabique) located in Ouagadougou and Bobo Dioulasso. However, despite all these national control efforts, rabies still occurring in both animal and human populations, remaining a major public health concern in the country (Savadogo et al., 2020).

To our knowledge, no major action have been implemented by the government and public health stakeholders to raise awareness within communities and promote adequate health behaviors regarding rabies control and 
prevention. This could be explained by a lack of documented data on knowledge, attitutes and practices of rabies in communities. However, many studied have indicated the relevance of stakeholders awareness in diseases prevention and control (Fusi Ngwa et al., 2010 ; Yandaï et al., 2017). To address this gap, our study was conducted in five urban and rural health districts with the aim of decribing the knowledge, attitudes and practices of people regarding rabies and its prevention and control.

\section{MATERIALS AND METHODS}

\section{Study area and sampling}

A cross-sectional house-to-house survey was conducted from November to December, 2019 with the objective of assessing people's knowledge, attitudes and practices regarding rabies. Up to the end of this research, rabies was not included in the list of priority animal diseases under surveillance (Foot and mouth disease, Newcastle disease, African swine fever, High pathogen avian influenza, Peste des petits ruminants, Bovine peripneumonia). In the absence of national statistical evidence on the size of dog population and rabies epidemiology in the different regions, the selection of study sites was made on the basis of logistic convenience (Aiyedun et Olugasa, 2012; Hergert et al., 2016, Savadogo et al., 2021) and guidance provided by animal health workers as follow: Bogodogo health district in the Centre region, Dano health district in the Sud-Ouest region, Dô health district in the Hauts-Bassins region and Garango and Tenkodogo health districts in the Centre-Est region (Figure 1 and Table 1). The survey included 320 households selected from the five health districts. In each health district, with regard to accessibility criteria, only households located along the main streets were surveyed as follow: on either side of a randomly selected street, every third household was considered and surveyed.

\section{Questionnaire interviews}

To capture all research objectives, a structured questionnaire (open and closedended questions) was developed to collect data through a face to face interviews. In each selected household, questions were asked to the head of household or any household member who agreed to participate. To ensure the data quality, interviewers were selected among local animal health workers who previously attended the UN-Food and Agriculture Organization (FAO) Field Epidemiology and Laboratory Training (FELT) and In-Service Applied Veterinary Epidemiology Training (ISAVET) Programs. Then, in each health district, interviews were conducted in a language undertandable to respondents (French, Mooré, Bissa, Dagara or Dioula).

As rabies is a zoonotic disease, this cross-sectional survey was conducted on the basis of the One Health two pilars: human subjects were household members, animal subjects were owned dogs. Based upon that, data collected on respondents and their households included variables like age, gender, level of study, position held in the household, type of housing, dog owning status of households, having observed or not any suspected rabid case, main information channels used and number of people per household. Dog characteristics included sex, age and purpose of keeping. Table 2 presents assessed variables in this particular research: knowledge level of rabies, perception on both animal and human rabies level of severity, attitudes and practices regarding rabies and its prevention and control. Thus, four questions were used to assess the knowledge level of rabies including vector animals, modes of transmission, description of the clinical signs and recommended prevention measures. For 
each question, scores (ranging from zero to three) were given according to the completeness and accuracy of answers (Table 2). Then, adding up the scores given to questions thus made it possible to rank the level of knowledge of rabies on a scale ranging from zero to nine : low if total score ranks from zero to two, medium if total score ranks from three to five and high if total score ranks from six to nine (Tables 3 and 4).

\section{Data analysis}

Data from all health districts were entered into a Microsoft Excel spreadsheet, and qualitative variables were scored. Then, the database was imported into $\mathrm{R}$ software version 3.6.1 (https://www.r-project.org/) and descriptive statistics were performed for proportions and means calculation. The associaton between categorial explanatory variables and dependent variables was assessed using Chi-square test with a significance level of $p<0.05$ as follow:

- Step 1: A first approach consisted in assessing the association between households' variables (health district in which it is located, area of location, gender, age, level of study, position held by respondent in the household, type of housing, dog owning status, previous history with rabies, main information channels used) and assessed variables decribing knowledge level of rabies, perceptions on animal rabies, perceptions on human rabies, attitudes towards rabies and practices with regard to bite cases management (Table 3).

- Step 2: In a second approach, researchers checked if the respondents' knowledge levels of rabies (low, medium, high) was associated with their perceptions, attitudes and practices towards rabies and the prevention measures (Table 4).

- Step 3: Finally, a third approach assessed the association between respondents' perceptions and attitudes with their practices regarding bite cases management (Table 5).

Using these three approaches aimed at identifying variables that could influence participants' knowledge of rabies, their attitudes, perceptions and practices towards this zoonosis respectively.

\section{Ethical considerations}

This research obtained ethics approval from the Research Ethical Committee of the Université Cheikh Anta Diop (Protocole0322/2018/CER/UCAD). The study proposal was approved by the Direction Générale des Services Vétérinaires (DGSV), Ministère des Ressources Animales et Halieutiques (MRAH) of Burkina Faso. Interviews were conducted by experienced animal health workers in each health district. Prior to the administration of the questionnaire, the interviewers explained the study background to each respondent and obtained verbal consent to conduct the interview. 
Table 1 : Study areas' sociogeographic description.

\begin{tabular}{|c|c|c|c|c|c|}
\hline $\begin{array}{l}\text { Characteristics of the four administrative } \\
\text { regions }\end{array}$ & Centre & Centre-Est & Hauts Bassins & Sud-Ouest & Total \\
\hline Localisation & $12^{\circ} 20^{\prime} \mathrm{N}, 1^{\circ} 30^{\prime} \mathrm{W}$ & $11^{\circ} 30^{\prime} \mathrm{N}, 0^{\circ} 15^{\prime} \mathrm{W}$ & $11^{\circ} 15^{\prime} \mathrm{N}, 4^{\circ} 30^{\prime} \mathrm{W}$ & $10^{\circ} 19^{\prime} \mathrm{N}, 3^{\circ} 10^{\prime} \mathrm{W}$ & - \\
\hline Size $\left(\mathrm{Km}^{2}\right)^{1}$ & 2,869 & 14,709 & 25,574 & 16,533 & 59,685 \\
\hline Number of provinces & 1 & 3 & 3 & 4 & 11 \\
\hline Number of health districts ${ }^{2}$ & 5 & 7 & 7 & 4 & 23 \\
\hline Districts included in the study ${ }^{2}$ & Bogodogo & $\begin{array}{l}\text { Garango and } \\
\text { Tenkodogo }\end{array}$ & Do & Dano & - \\
\hline Surveyed sites & Saaba & $\begin{array}{l}\text { Garango and } \\
\text { Tenkodogo }\end{array}$ & Bobo Dioulasso & $\begin{array}{l}\text { Dano, Dissin and } \\
\text { Ouessa }\end{array}$ & - \\
\hline Estimated population size $^{1}$ & $2,231,807$ & $1,343,079$ & $1,776,803$ & 729,362 & $6,081,051$ \\
\hline
\end{tabular}

Table 2 : Description of variables used to assess participants' knowledge, attitudes and practices regarding rabies prevention and control.

\begin{tabular}{ll}
\hline Variables & Example of answers given by respondents \\
\hline Animals that can transmit rabies & \\
\hline Incorrect answer & Do not know, birds, snake, scorpion \\
\hline One animal named & Dog, cat, donkey, horse, monkey, goat, sheep, rondent, pig, wild mammals \\
\hline Two animals named & Dog, cat, donkey, horse, monkey, goat, sheep, rondent, pig, wild mammals \\
\hline At least three animals named & Dog, cat, donkey, horse, monkey, goat, sheep, rondent, pig, wild mammals \\
\hline Mode of transmission of rabies & \\
\hline Incorrect answer & Do not know, contaminated food or water \\
\hline
\end{tabular}


M. SAVADOGO et al. / Int. J. Biol. Chem. Sci. 15(5): 1800-1816, 2021

\begin{tabular}{|c|c|c|}
\hline One mode of transmission named & Bite, scratch, saliva, licking on a wound & 1 \\
\hline Two modes of transmission named & Bite, scratch, saliva, licking on a wound & 2 \\
\hline At least three modes of transmission named & Bite, scratch, saliva, licking on a wound & 3 \\
\hline \multicolumn{3}{|l|}{ Description of rabies' clinical signs } \\
\hline Incorrectly described & No correct clinical sign named & 0 \\
\hline One clinical sign described & Agressivity, paralysis, hypersalivation, non-recognition of owner, anorexia & 1 \\
\hline Two clinical signs described & Agressivity, paralysis, hypersalivation, non-recognition of owner, anorexia & 2 \\
\hline At least three clinical signs described & Agressivity, paralysis, hypersalivation, non-recognition of owner, anorexia & 3 \\
\hline \multicolumn{3}{|l|}{ Animal rabies prevention measures } \\
\hline Uncorrect answer & Do not know, culling dogs, & 0 \\
\hline Correct answer & Vaccinating against rabies, confining dogs, providing veterinary care & 1 \\
\hline \multicolumn{3}{|c|}{ Perception on rabies severity level (both for animal and human) } \\
\hline Minor disease & - & - \\
\hline Severe disease & - & - \\
\hline Fatal disease & - & - \\
\hline \multicolumn{3}{|l|}{ Attitude towards suspected rabid animal } \\
\hline Negative attitude & Culling suspected dog, no response, nothing done & - \\
\hline Positive attitude & Calling a veterinarian, isolating the animal, alerting local authorities & - \\
\hline \multicolumn{3}{|l|}{ Practices in case of a bite } \\
\hline Uncorrect & $\begin{array}{l}\text { Nothing done, traditional treatment, culling biting animal, applying biting animal's } \\
\text { hair on the wound }\end{array}$ & - \\
\hline Correct & $\begin{array}{l}\text { Washing the wound with water and soap, look for postexposition vaccination, } 15 \\
\text { days vet observation of biting animal }\end{array}$ & - \\
\hline
\end{tabular}




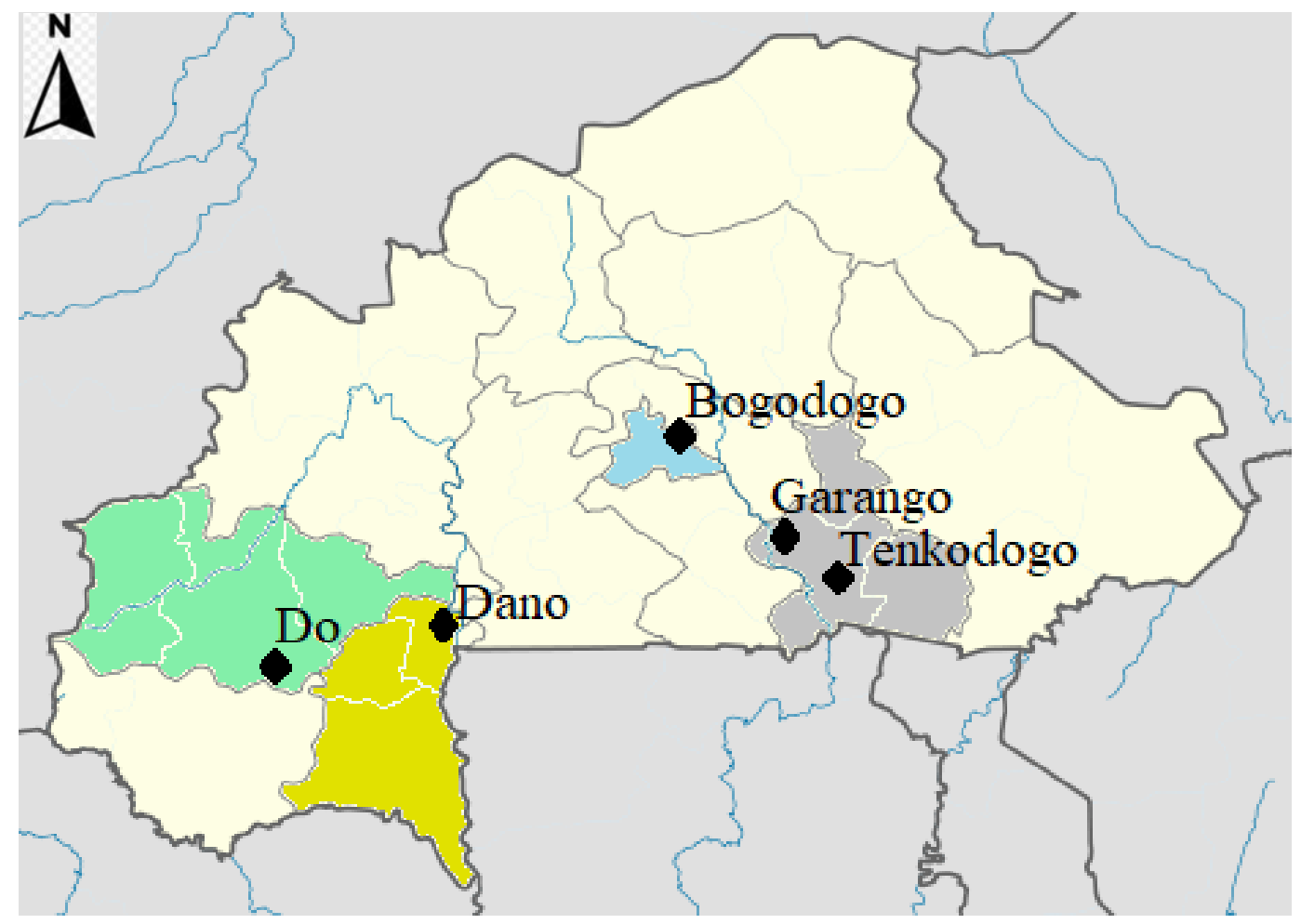

Figure 1: Location of health districts in which households were surveyed in this study. Green : Hauts Bassins region; Yellow : Sud-Ouest region; Blue : Centre region; Grey : Centre-Est region.

\section{RESULTS}

\section{Households' information}

The majority of surveyed households were located in urban area $(62.2 \%)$ (Table 3). Most of respondents were heads of households (66.9\%) and male (80.9\%). In terms of their education levels, most of them had studied $(65.6 \%)$. Almost all of surveyed households were described by participants as their permanent residence $(93.4 \%)$. In total, 2,251 persons were recorded in surveyed households with $46.6 \%$ of children not older than 15 years. From the 320 households, $57.2 \%$ owned at least a dog with $373 \mathrm{dogs}$ recorded during the survey, with a male per female ratio of $2.3: 1$ and dog per household ratio of $11.6: 10$. In the visited health districts, dog owning households percentage ranked from $34.2 \%$ (in Bogodogo) to $91.7 \%$ (in Dano). Households owned dogs for various socio-economic purposes, including home security $(89.1 \%)$ and companionship (10.9\%). For information needs, households used radio $(50.3 \%)$ and television (43.7\%) more than online social media (4.1\%) and newspaper (1.9\%).

\section{Participants' knowledge of rabies}

Concerning animals that can transmit rabies to human, 51.6\% (165/320) and 32.9\% $(105 / 320)$ of respondents knew that dog and cat were known to be rabies vectors, respectively. About $67.5 \%$ (216/320) of respondents knew that bite was a mode of transmission of rabies while $52.6 \%(168 / 320)$ and $28.5 \%$ (91/320) described agressivity and paralysis, respectively, as rabies major clinical signs. When asked about animal rabies control measures, $89.7 \%$ (287/320) of respondents reported that animal should be vaccinated against rabies. In Table 3 knowledge levels of 
rabies are presented by health district, household location, age, gender, level of study, position in the household, type of housing, dog owning status, previous history with rabies and main information channels used by households. Among investigated sociodemographic characteristics, health district, area where household was located, age of respondents, their level of study, their previous history with rabies and main information channels they used were significantly associated with knowledge level of rabies $(\mathrm{p}<0.05)$. The oldest respondents, those living in urban areas, those having the highest levels of study, those having a previous history with rabies and those using radio and television as information channels were more likely to be well aware of rabies.

\section{Perceptions and attitudes towards rabies}

Rabies was perceived by respondents as minor disease $(2.1 \%)$, severe disease $(17.2 \%)$ and fatal disease $(80.6 \%)$ both for animal and human (Table 5). Perceptions on animal rabies were significantly associated with health district, area where household was located, type of housing, household dog owning status, age of respondents, their position in households, their previous history with rabies $(\mathrm{p}<0.05)$. Only three socio-demographic variables (health district, household dog owning status, previous history with rabies) were significantly associated with the perception of human rabies $(\mathrm{p}<0.05)$. Animal rabies and human rabies were more likely to be perceived as fatal disease by participants with higher levels of knowledge. When questionned about their attitudes with regards to a suspected rabid animal, most of respondents (76.4\%) reported that they would kill the animal whereas about $20.8 \%$ would call the local animal health worker or any other local authority. Attitude towards rabies (negative or positive) was associated with the level of education, the area where household was located and the health district $(\mathrm{p}<0.05)$. In addition, Table 4 shows that perceptions on animal rabies, perceptions of human rabies as well as attitudes towards rabies were significantly associated with knowledge level of rabies $(\mathrm{p}<0.05)$.

\section{Practices in terms of health seeking behaviours}

When asked about actions to be initiated following any bite caused by a suspected rabid animal, most of respondents reported that they would seek for postexposure prophylaxis $(45.7 \%, 137 / 320)$ while $9.4 \%$ (30/320) of them reported that they would apply traditional treatment (for example applying the biting animal hair on the wound). However, $41.6 \%$ (133/320) of respondents reported that they would not seek for any treatment. Two sociodemographic variables (health district of respondents and their previous history with rabies) showed a significant association with the people practices (uncorrect or correct) in case of a bite by any suspected rabid animal $(\mathrm{p}<0.05)$. Following a bite, health seeking behaviors were more likely to be uncorrect in respondents with no previous history with rabies and those living in Dano and Do health districts. In addition, people health seeking behaviors were significantly associated with their levels of knowledge regarding rabies, their perceptions and attitudes towards the disease both in animal and human $(\mathrm{p}<0.05)$ (Tables 4 and 5). 
M. SAVADOGO et al. / Int. J. Biol. Chem. Sci. 15(5): 1800-1816, 2021

Table 3 : Association between knowlege, perceptions, attitude and practices of rabies and socio-demographic characteristics of the respondents in the study area.

\begin{tabular}{|c|c|c|c|c|c|c|c|c|c|c|c|c|c|c|c|c|c|c|}
\hline \multirow[t]{2}{*}{ Variables } & \multicolumn{3}{|c|}{$\begin{array}{c}\text { Knowledge level of } \\
\text { rabies }\end{array}$} & \multirow[t]{2}{*}{$\begin{array}{c}\text { p- } \\
\text { value }\end{array}$} & \multicolumn{3}{|c|}{$\begin{array}{c}\text { Perception on animal } \\
\text { rabies }\end{array}$} & \multirow[t]{2}{*}{$\begin{array}{l}\text { p- } \\
\text { value }\end{array}$} & \multicolumn{3}{|c|}{$\begin{array}{c}\text { Perception on human } \\
\text { rabies }\end{array}$} & \multirow[t]{2}{*}{$\begin{array}{l}p- \\
\text { value }\end{array}$} & \multicolumn{2}{|c|}{$\begin{array}{c}\text { Attitude towards } \\
\text { rabies }\end{array}$} & \multirow[t]{2}{*}{$\begin{array}{l}\text { p- } \\
\text { value }\end{array}$} & \multicolumn{2}{|c|}{$\begin{array}{c}\text { Practices in case of } \\
\text { a bite }\end{array}$} & \multirow[t]{2}{*}{ p-value } \\
\hline & $\begin{array}{l}\text { Low } \\
(\%)\end{array}$ & $\begin{array}{c}\text { Medium } \\
(\%)\end{array}$ & $\begin{array}{c}\text { High } \\
(\%)\end{array}$ & & $\begin{array}{l}\begin{array}{l}\text { Minor } \\
\text { disease } \\
(\%)\end{array} \\
\end{array}$ & $\begin{array}{l}\begin{array}{l}\text { Severe } \\
\text { disease } \\
(\%)\end{array} \\
\end{array}$ & $\begin{array}{l}\text { Fatal } \\
\text { disease } \\
(\%)\end{array}$ & & $\begin{array}{l}\text { Minor } \\
\text { disease } \\
(\%)\end{array}$ & $\begin{array}{l}\text { Severe } \\
\text { disease } \\
(\%)\end{array}$ & $\begin{array}{l}\text { Fatal } \\
\text { disease } \\
(\%) \\
\end{array}$ & & $\begin{array}{l}\text { Negative } \\
(\%)\end{array}$ & $\begin{array}{l}\text { Positive } \\
(\%)\end{array}$ & & $\begin{array}{l}\text { Uncorrect } \\
(\%)\end{array}$ & $\begin{array}{l}\text { Correct } \\
(\%)\end{array}$ & \\
\hline \multicolumn{19}{|c|}{ Belonging health district } \\
\hline $\begin{array}{l}\text { Bogodogo } \\
(\mathrm{n}=111)\end{array}$ & 15.3 & 56.8 & 27.9 & \multirow{5}{*}{0.03} & 0.9 & 34.2 & 65.8 & \multirow{5}{*}{0.00} & 0.0 & 29.7 & 70.3 & \multirow{5}{*}{0.00} & 99.1 & 0.9 & \multirow{5}{*}{0.00} & 57.7 & 42.3 & \multirow{5}{*}{0.00} \\
\hline $\begin{array}{l}\text { Dano } \\
(\mathrm{n}=60)\end{array}$ & 23.4 & 48.3 & 28.3 & & 1.7 & 5.0 & 93.3 & & 0.0 & 8.3 & 91.7 & & 85.0 & 15.0 & & 75.0 & 25.0 & \\
\hline Do $(\mathrm{n}=51)$ & 21.6 & 33.3 & 45.1 & & 2.0 & 7.8 & 90.2 & & 9.8 & 11.8 & 78.4 & & 84.3 & 15.7 & & 76.5 & 23.5 & \\
\hline $\begin{array}{l}\text { Garango } \\
(\mathrm{n}=48)\end{array}$ & 16.7 & 33.3 & 50.0 & & 4.2 & 14.6 & 81.3 & & 0.0 & 12.5 & 87.5 & & 83.3 & 16.7 & & 54.2 & 45.8 & \\
\hline $\begin{array}{l}\text { Tenkodogo } \\
(\mathrm{n}=50)\end{array}$ & 16.0 & 38.0 & 46.0 & & 4.0 & 8.0 & 88.0 & & 4.0 & 10.0 & 86.0 & & 72.0 & 28.0 & & 28.0 & 72.0 & \\
\hline \multicolumn{19}{|c|}{ Area where household was located } \\
\hline $\begin{array}{l}\text { Urbain } \\
(\mathrm{n}=199)\end{array}$ & 14.1 & 39.2 & 46.7 & \multirow{2}{*}{0.00} & 0.5 & 15.1 & 84.4 & \multirow{2}{*}{-0.03} & 3.5 & 18.1 & 78.4 & \multirow{2}{*}{0.09} & 84.4 & 15.6 & \multirow{2}{*}{0.03} & 60.8 & 39.2 & \multirow{2}{*}{0.34} \\
\hline $\begin{array}{l}\text { Periurbain } \\
(\mathrm{n}=121)\end{array}$ & 24.8 & 54.5 & 20.7 & & 5.0 & 20.7 & 74.4 & & 0.0 & 15.7 & 84.3 & & 92.6 & 7.4 & & 55.4 & 44.6 & \\
\hline \multicolumn{19}{|l|}{$\begin{array}{l}\text { Age (years) } \\
\end{array}$} \\
\hline $\begin{array}{l}<18 \\
(\mathrm{n}=11)\end{array}$ & 45.5 & 9.0 & 45.5 & \multirow[b]{2}{*}{0.02} & 18.2 & 18.2 & 63.6 & \multirow[b]{2}{*}{0.00} & 2.3 & 16.8 & 96.5 & \multirow[b]{2}{*}{0.79} & 81.8 & 18.2 & \multirow[b]{2}{*}{0.56} & 45.5 & 54.5 & \\
\hline $\begin{array}{l}18 \text { and } \\
\text { older } \\
(n=309)\end{array}$ & 17.2 & 46.3 & 36.5 & & 1.6 & 17.2 & 81.2 & & 0.0 & 27.3 & 72.7 & & 87.7 & 12.3 & & 59.2 & 40.8 & 0.36 \\
\hline
\end{tabular}


M. SAVADOGO et al. / Int. J. Biol. Chem. Sci. 15(5): 1800-1816, 2021

\section{Gender}

Female

$(\mathrm{n}=61)$

Male

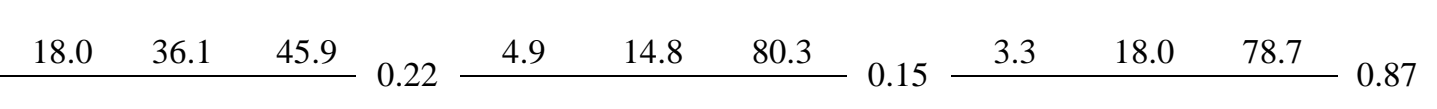

\begin{tabular}{ccccc}
91.8 & 8.2 & & 62.3 & 37.7 \\
\cline { 1 - 2 } \cline { 4 - 5 } 06.5 & 13.5 & & 57.9 & 42.1
\end{tabular}

\section{Position held in the household}

Head of

household

$(\mathrm{n}=214)$

\begin{tabular}{lll}
15.0 & $46.3 \quad 38.8$ \\
\hline
\end{tabular}

\begin{tabular}{lll}
$0.9 \quad 14.0 \quad 85.0$ \\
\hline
\end{tabular}

$1.4 \quad 14.0 \quad 84.6$

\begin{tabular}{l}
$85.0 \quad 15.0$ \\
\hline
\end{tabular}

\begin{tabular}{ll}
55.6 & 44.4 \\
\hline
\end{tabular}

head of

household

0.08

0.00

0.15

92.7

7.3

0.17

$(n=41)$ $\begin{array}{lll}24.4 & 31.7 \quad 43.9\end{array}$

$\begin{array}{lll}7.3 & 12.2 & 80.5\end{array}$

\begin{tabular}{lll}
4.9 & $17.1 \quad 78.0$ \\
\hline
\end{tabular}

member of

household

$(n=65)$

$24.6 \quad 49.2 \quad 26.2$

$\begin{array}{lll}3.1 & 30.8 & 66.2\end{array}$

92.3

7.7

\section{Level of education}

\begin{tabular}{|c|c|c|c|c|c|c|c|c|c|c|c|c|c|c|c|c|c|c|}
\hline $\begin{array}{l}\text { Illetrate } \\
(n=109)\end{array}$ & 28.4 & 50.5 & 21.1 & \multirow{4}{*}{0.00} & 4.6 & 16.5 & 78.9 & \multirow{4}{*}{0.26} & 4.6 & 19.3 & 76.1 & \multirow{4}{*}{0.41} & 93.6 & 6.4 & \multirow{4}{*}{0.03} & 63.3 & 36.7 & \multirow{2}{*}{0.07} \\
\hline $\begin{array}{l}\text { Primary } \\
(\mathrm{n}=78)\end{array}$ & 21.8 & 55.1 & 23.1 & & 2.6 & 16.7 & 80.8 & & 1.3 & 16.7 & 82.1 & & 89.7 & 10.3 & & 64.1 & 35.9 & \\
\hline $\begin{array}{l}\text { Secondary } \\
(\mathrm{n}=95)\end{array}$ & 8.4 & 33.7 & 57.9 & & 0.0 & 21.1 & 78.9 & & 1.1 & 14.7 & 84.2 & & 83.2 & 16.8 & & 55.8 & 44.2 & \\
\hline $\begin{array}{l}\text { University } \\
(\mathrm{n}=37)\end{array}$ & 5.4 & 35.1 & 59.5 & & 0.0 & 8.1 & 91.9 & & 0.0 & 16.2 & 83.8 & & 75.7 & 24.3 & & 40.5 & 59.5 & \\
\hline
\end{tabular}

$(n=37)$ 
M. SAVADOGO et al. / Int. J. Biol. Chem. Sci. 15(5): 1800-1816, 2021

\begin{tabular}{|c|c|c|c|c|c|c|c|c|c|c|c|c|c|c|c|c|c|c|}
\hline \multicolumn{19}{|c|}{ Type of housing } \\
\hline $\begin{array}{l}\text { Collective } \\
(\mathrm{n}=66)\end{array}$ & 22.7 & 43.9 & 33.4 & \multirow[b]{2}{*}{0.53} & 6.1 & 16.7 & 77.3 & & 3.0 & 16.7 & 80.3 & \multirow{2}{*}{0.91} & 89.4 & 10.6 & \multirow{2}{*}{0.60} & 62.1 & 37.9 & \multirow{2}{*}{0.53} \\
\hline $\begin{array}{l}\text { Individual } \\
(\mathrm{n}=254)\end{array}$ & 16.9 & 45.3 & 37.8 & & 1.2 & 17.3 & 81.5 & 0.04 & 2.0 & 17.3 & 80.7 & & 87.0 & 13.0 & & 57.9 & 42.1 & \\
\hline
\end{tabular}

\section{Presence or not of dog in the household (dog owning status)}

\begin{tabular}{|c|c|c|c|c|c|c|c|c|c|c|c|c|c|c|c|c|c|c|}
\hline $\begin{array}{l}\text { No } \\
(n=137)\end{array}$ & 20.4 & 46.0 & 33.6 & \multirow{2}{*}{0.48} & 2.2 & 24.8 & 73.0 & 001 & 4.4 & 24.1 & 71.5 & \multirow{2}{*}{0.00} & 90.5 & 9.5 & \multirow{2}{*}{0.16} & 61.3 & 38.7 & \multirow{2}{*}{0.42} \\
\hline $\begin{array}{l}\text { Yes } \\
(n=183)\end{array}$ & 16.4 & 44.3 & 39.3 & & 2.2 & 11.5 & 86.3 & 0.01 & 0.5 & 12.0 & 87.4 & & 85.2 & 14.8 & & 56.8 & 43.2 & \\
\hline
\end{tabular}

\section{Having observed or not any suspected rabid case (previous history with rabies)}

\begin{tabular}{|c|c|c|c|c|c|c|c|c|c|c|c|c|c|c|c|c|c|c|}
\hline $\begin{array}{l}\text { No } \\
(n=131)\end{array}$ & 30.5 & 40.5 & 29.0 & \multirow{2}{*}{0.00} & 5.3 & 22.9 & 71.8 & 00 & 4.6 & 22.9 & 72.5 & \multirow{2}{*}{0.01} & 87.0 & 13.0 & \multirow{2}{*}{0.83} & 68.7 & 31.3 & \multirow{2}{*}{0.00} \\
\hline $\begin{array}{l}\text { Yes } \\
(n=189)\end{array}$ & 9.5 & 48.2 & 42.3 & & 0.0 & 13.2 & 86.8 & 0.00 & 0.5 & 13.2 & 86.2 & & 87.8 & 12.2 & & 51.9 & 48.1 & \\
\hline
\end{tabular}

\section{Main information channels used in the household}

\begin{tabular}{|c|c|c|c|c|c|c|c|c|c|c|c|c|c|c|c|c|c|c|}
\hline $\begin{array}{l}\text { Internet } \\
(n=13)\end{array}$ & 7.7 & 30.8 & 61.5 & \multirow{4}{*}{0.00} & 0.0 & 15.4 & 84.6 & \multirow{4}{*}{0.99} & 0.0 & 15.4 & 84.6 & \multirow{4}{*}{0.99} & 84.6 & 15.4 & \multirow{4}{*}{0.43} & 46.2 & 53.8 & \multirow{4}{*}{0.32} \\
\hline $\begin{array}{l}\text { Newspaper } \\
(n=6)\end{array}$ & 0.0 & 50.0 & 50.0 & & 0.0 & 16.7 & 83.3 & & 0.0 & 16.7 & 83.3 & & 100.0 & 0.0 & & 33.3 & 66.7 & \\
\hline $\begin{array}{l}\text { Radio } \\
(n=161)\end{array}$ & 21.1 & 55.9 & 23.0 & & 3.7 & 16.8 & 79.5 & & 1.2 & 18.0 & 80.7 & & 90.7 & 9.3 & & 62.7 & 37.3 & \\
\hline $\begin{array}{l}\text { TV } \\
(n=140)\end{array}$ & 16.4 & 33.6 & 50.0 & & 0.7 & 17.9 & 81.4 & & 3.6 & 16.4 & 80.0 & & 83.6 & 16.4 & & 56.4 & 43.6 & \\
\hline
\end{tabular}


Table 4 : Association between respondents' perceptions, attitude and practices of rabies prevention and their knowledge levels of rabies.

\begin{tabular}{|c|c|c|c|c|c|c|c|c|c|c|c|c|c|c|}
\hline \multirow[t]{2}{*}{ Variables } & \multicolumn{3}{|c|}{$\begin{array}{l}\text { Perception on } \\
\text { animal rabies }\end{array}$} & \multirow[t]{2}{*}{$\begin{array}{l}\text { p- } \\
\text { value }\end{array}$} & \multicolumn{3}{|c|}{$\begin{array}{l}\text { Perception on human } \\
\text { rabies }\end{array}$} & \multirow[t]{2}{*}{$\begin{array}{l}p- \\
\text { value }\end{array}$} & \multicolumn{2}{|c|}{ Attitude towards rabies } & \multirow[t]{2}{*}{$\begin{array}{l}p- \\
\text { value }\end{array}$} & \multicolumn{2}{|c|}{$\begin{array}{l}\text { Practices in case of a } \\
\text { bite }\end{array}$} & \multirow[t]{2}{*}{$\begin{array}{l}\text { p- } \\
\text { value }\end{array}$} \\
\hline & $\begin{array}{l}\text { Minor } \\
\text { disease } \\
(\%)\end{array}$ & $\begin{array}{l}\text { Severe } \\
\text { disease } \\
(\%)\end{array}$ & $\begin{array}{l}\text { Fatal } \\
\text { disease } \\
(\%)\end{array}$ & & $\begin{array}{l}\text { Minor } \\
\text { disease } \\
(\%)\end{array}$ & $\begin{array}{l}\text { Severe } \\
\text { disease } \\
(\%)\end{array}$ & $\begin{array}{l}\text { Fatal } \\
\text { disease } \\
(\%)\end{array}$ & & Negative (\%) & $\begin{array}{l}\text { Positive } \\
(\%)\end{array}$ & & $\begin{array}{l}\text { Uncorrect } \\
(\%)\end{array}$ & $\begin{array}{l}\text { Correct } \\
(\%)\end{array}$ & \\
\hline \multicolumn{15}{|c|}{ Knowledge level of rabies } \\
\hline Low $(n=58)$ & 12.1 & 22.4 & 65.5 & \multirow{3}{*}{0.00} & 8.6 & 20.7 & 70.7 & \multirow{3}{*}{0.00} & 91.4 & 8.6 & \multirow{3}{*}{0.04} & 84.5 & 15.5 & \multirow{3}{*}{0.00} \\
\hline $\begin{array}{l}\text { Medium } \\
(\mathrm{n}=144)\end{array}$ & 0.0 & 20.8 & 79.2 & & 1.4 & 22.2 & 76.4 & & 91.0 & 9.0 & & 56.9 & 43.1 & \\
\hline $\operatorname{High}(\mathrm{n}=118)$ & 0.0 & 10.2 & 89.8 & & 0.0 & 9.3 & 90.7 & & 81.4 & 18.6 & & 48.3 & 51.7 & \\
\hline
\end{tabular}

Table 5 : Association between respondents' health practices and their perceptions and attitude with regard to rabies.

\begin{tabular}{|c|c|c|c|}
\hline \multirow[b]{2}{*}{ Variables } & \multicolumn{2}{|c|}{ Practices in case of a bite } & \multirow[b]{2}{*}{ p-value } \\
\hline & Uncorrect (\%) & Correct (\%) & \\
\hline \multicolumn{4}{|c|}{ Perception on animal rabies } \\
\hline Minor disease $(n=7)$ & 100.0 & 0.0 & \multirow{3}{*}{0.00} \\
\hline Severe disease $(n=55)$ & 74.5 & 25.5 & \\
\hline Fatal disease $(n=258)$ & 54.3 & 45.7 & \\
\hline \multicolumn{4}{|c|}{ Perception on human rabies } \\
\hline Minor disease $(n=7)$ & 100.0 & 0.0 & \multirow{2}{*}{0.00} \\
\hline Severe disease $(n=55)$ & 70.9 & 29.1 & \\
\hline Fatal disease $(\mathrm{n}=258)$ & 55.0 & 45.0 & \\
\hline \multicolumn{4}{|c|}{ Attitude towards rabies } \\
\hline Negative $(n=280)$ & 61.1 & 38.9 & 0.02 \\
\hline
\end{tabular}




\section{DISCUSSION}

During last decades, many studies proved that rabies can be eliminated in low and middle income countries (Cleaveland et al., 2003 ; Lembo et al., 2010 ; Zinsstag et al., 2011 ; Vigilato et al., 2013). Nowadays, the feasibility of rabies elimination worldwide is recognized by the key international health organizations led by the World Animal Health Organization (OIE), the World Health Organization (WHO), the Food and Agriculture Organization (FAO) and the Global Alliance for Rabies Control (GARC). This international high level commitment led to the launch of the United Against Rabies (UAR) plateform in September, 2020. Beyond medical and veterinary agencies, the implementation of rabies control and prevention strategies towards the 2030 global objective of zero human deaths, requires effective participation of all stakeholders, including at community level. With this in mind, it is important to assess whether community members is aware about rabies and prevention measures. Thus, identifying and filling the general public knowledge and behaviours gaps could help to ensure effectiveness in field interventions (Mama Cissé et al., 2020 ; Mosimann et al., 2017 ; Castillo-Neyra et al., 2017).

The study showed that most of households owned a least a dog (dog per household ratio was 11.6:10) highlighting the socio-economic importance of dogs within communities, including guarding, companionship, hunting and as meat sources (Aidedun et Olugasa, 2012 ; Jibart et al., 2015 ; Dahourou et al., 2021). Meanwhile, dogs are known to be the main rabies vector animal in African countries (Jibart et al., 2015; Savadogo et al., 2020). The findings also indicated that most of participants were aware of rabies as a disease transmitted through rabid animal bite. Nevertheless, studies conducted in Tanzania and Chad obtained higher proportions of participants who had knowledge of rabies and recommended control measures (Sambo et al., 2014 ; Mindekem et al., 2017). The knowledge levels were significantly higher in the oldest participants and those living in urban areas, indicating that the risk of developing rabies may be higher for young persons and poor communities (Dodet et al., 2010). The significant association between participants' study levels, used information channels and their knowlegde levels with regard to rabies is a reminder of the key role of education and media in public health awareness strategy (Ahmed et al., 2014 ; Thys et al., 2019). Thus, in poor communities with low or no access to formal education, the risk of transmission of rabies to people could be higher (Sambo et al., 2014). The particular usefulness of radio and television, in coveying health key messages in rural communities, was previously reported by Hairi et al. (2003) in Kuala Kangsar. Finally, the results highlight the need of awareness raising efforts in order to address knowledge gaps, paying a particular attention to communities living in remote areas (Castillo-Neyra et al., 2017 ; Savadogo et al., 2020).

Animal and human rabies was perceived by most of participants as fatal disease after the onset of the clinical signs. This observation raises the question of why a significant proportion of people (51\%) claimed that they would not seek for postexposure vaccination if their were bitten by suspected rabid animal. Indeed, many studies descibed lower PEP seeking in exposed communities (Sambo et al., 2014 ; Mindekem et al., 2017 ; Hampson et al., 2008). Sondo et al. (2014), reporting that constraints such as the distance to the health services and the high cost of vaccine impede the accessibility of community members to PEP. Concerning the attitudes towards rabies control, most of respondents appeared to have negative attitudes, despite that in Burkinabe culture, interviwees used to answer yes in order to please to interviewers. In fact, when 15 days veterinary observation is recommended for biting animals, the majority of participants reported that they would immediately kill biting dogs. In the context of Burkina Faso, where owned dogs are often roaming, properly vaccinated biting dogs could be killed by the communities. A such inadequate practice may contribute discouraging owners who are not well aware, 
from vaccinating their dogs against rabies. Beyond the socio-demographic characteristics, participants who had low and medium knowledge levels were more likely to develop negative attitude towards rabies, consisting in killing biting dogs, low reporting of bite cases to veterinary or medical services (Mosimann et al., 2017 ; Savadogo et al., 2021). This result indicates that unadequate attitudes regarding bite cases management are a point to be addressed in educational aspects of rabies control planning.

Most of participants were aware that rabies is a fatal disease and transmitted to human through suspected rabid animal's bite. However, low proportion of participants claimed to have correct practices in case of bite, indicating the need to pay more attention to the practices aspects in any awareness effort. Similar findings were reported by Hairi et al. (2003) in Malaysia, Sambo et al. (2014) in Tanzania, Mindekem et al. (2017) in Chad, Ali et al. (2013) in Ethiopia and Rumana et al. (2013) in Bangladesh. Respondents with no previous history with rabies were more likely to adopt uncorrect health practices. This result could be explained by the lack of regular rabies interventions in the study areas, involving and educating communities, as reported by Ojieabu et al. (2011). Indeed, Burkina Faso has never developed a rabies control program (Dodet et al., 2010). Therefore, field activities such as rabies outbreak investigations and mass dog vaccination also help to popularize rabiesrelated information in an interactive way. Only two socio-demographic factors (health district of belonging and previous history with rabies) were significantly associated with practices towards rabies suggesting that in the communities, health practices are mainly affected by local culture and some traditional practices. Moreover, participants' health seeking practices were significantly associated with their knowledge levels, perceptions and attitudes regarding rabies and prevention methods. In contrast to results obtained by Hairi et al. (2003) in Malaysia, our findings show that improved knowledge levels of populations could lead to better attitudes and practices towards rabies control and prevention.

Although rabies is endemic in Burkina Faso, no national and integrated rabies control strategy has been developed in the country. The scarcity of rabies control field interventions could possibly explained the inadequate kowledge, attitudes and practices regarding rabies and its prevention and control measures. Therefore, all national rabies control involvedstakeholders should take the opportunity of the ongoing international high level commitment towards zero human case by 2030, to develop and implement a transdisciplinary rabies control program, fostering effective collaboration between all stakeholders.

\section{Conclusion}

Good knowledge, attitudes and practices regarding rabies and its prevention and control are important moving towards global zero by 2030 objective. The study showed that community members were not well aware of rabies in a satisfactory manner. Therefore, rabies control stakeholders should address the knowledge gaps through mass awareness raising campaigns, providing key messages regarding the risks of contamination, the recommended practices to prevent avoidable deaths. Therefore, professionals and decision-makers should be trained to give appropriate messages aiming to change certain deeply ingrained traditional practices including traditional treatment seeking, dog roaming and non-vaccination of owned dogs.

\section{COMPETING INTERESTS}

The authors of this manuscript declare that there is no competing interest related to it.

\section{AUTHORS' CONTRIBUTIONS}

MS: Conceptualization, Methodology, Investigation, Supervision, Formal analysis, Writing Original draft; LDD: Methodology, Supervision, Formal analysis, Writing Original draft; EK and AKT: Supervision, Project administration, Funding acquisition, Original draft preparation; HVG and JS: Validation, Supervision; SHO, ASO and GHB: 
Investigation; RBA and ZT: Validation, Supervision, Writing, Review and Editing.

\section{ACKNOWLEDGMENTS}

The authors want to acknowledge all families and adminstratives authorities who agreed to collaborate and welcome interviewers during data collection. We are grateful to Dr. Ismaïla Seck who help us in the methodology and reviewing the manuscript, and Mr. Zakaria Souli for his great support for statistical analysis. Field investigation was made possible by technical and logistical support from the FAO-Emergency Center for Transboundary Animal Diseases (FAOECTAD), Ouagadougou, Burkina Faso.

\section{REFERENCES}

Acharya KP, Acharya N, Phuyal S, Upadhyaya M, Lasee S. 2020. One-health approach: A best possible way to control rabies. One Health, 10: 1001-1061. DOI : https://doi.org/10.1016/j.onehlt.2020.100 161.

Ahmed VO, Dzikwi AA, Umoh JU. 2014. Assessment of Knowledge, Attitude and Practice of Dog Owners to Canine Rabies in Wukari Metropolis, Taraba State Nigeria. Global J. Health Sci., 6: 226240.

DOI : https://doi.org/10.5539/gjhs.v6n5p226

Aiyedun JO, Olugasa BO. 2012. Identification and analysis of dog use, management practices and implications for rabies control in Ilorin, Nigeria. Sokoto J. Vet. Sci., $\quad \mathbf{1 0}(2) . \quad$ DOI : http://dx.doi.org/10.4314/sokjvs.v10i2.1

Ali A, Ahmed EY, Sifer DA. 2013. Study on Knowledge, Attitude and Practice of rabies among residents in Addis Ababa, Ethiopia. Ethiop. Vet. J., 17(2): 19-35. DOI : https://doi.org/10.4314/evj.v17i2.2

Burkina Faso. 2018. Décret N ${ }^{\circ} 2018$ 0730/PRES/PM/MRAH/MINEFID/MA TD/MSECU/MCIA portant réglementation de la police zoosanitaire au Burkina Faso.

Castillo-Neyra R, Brown J, Borrini K, Arevalo C, Levy MZ, Buttenheim A, Hunter GC, Becerra V, Behrman J, Paz-Soldan VA. 2017. Barriers to dog rabies vaccination during an urban rabies outbreak: Qualitative findings from Arequipa, Peru. PLoS Negl. Trop. Dis., 11(3): e0005460. DOI :

https://doi.org/10.1371/journal.pntd.0005 460

Cisse IM, Alassani A, Adjobimey M, Mikponhoue R, Hinsou AV, Ayelo P. 2020. Facteurs comportementaux et environnementaux associés au paludisme à Tourou (Bénin) en période de faible endémicité. Int. J. Biol. Chem. Sci., 14(8): 2737-2745.

DOI: https://doi.org/10.4314/ijbcs.v14i8.7

Cleaveland S, Kaare M, Tiringa P, Mlengeya T, Barrat J. 2003. A dog rabies vaccination campaign in rural Africa: impact on the incidence of dog rabies and human dog-bite injuries. Vaccine, 21(19): 65-73.

DOI:

https://doi.org/10.1016/S0264410X(02)00778-8

Dahourou LD, Savadogo M, Kaboré BA, Konaté A, Zerbo M, Guigma HV, Ouoba LB, Ouandaogo SH, Zerbo LH, Traoré A. 2021. Dog ownership, demographics, owners' knowledge of rabies, and factors associated with canine rabies vaccination in urban and rural areas of Dedougou, Burkina Faso. Vet. Anim. Sci., 14 : $100205 . \quad$ DOI : https://doi.org/10.1016/j.vas.2021.10020 5

Dodet B, le Bureau d'experts de la rage du continent africain (AfroREB), Adjogoua EV, Aguemon AR, Baba BA, Bara Adda S, Boumandouki P, Bourhy H, Brahimi M, Briggs D, Diallo MK, Diarra L, Diop B, Diop SAG, Fesriry B, Gosseye S, Kharmachi H, Le Roux K, Nakoune Yandoko E, Nel L, Ngome J-M, Nzengue E, II Ramahefalalao EF, Ratsitorahina M, Rich H, Simpore L, Soufi A, Tejiokem MC, Thiombiano R, Tiembre I, Traore AK, Wateba MI, Yahaye H., Zaouia I. 2010. The fight against rabies in Africa : from recognition to action. Bull. Soc. Pathol. Exot., 103(1): 51-59. DOI : https://doi.org/10.1007/s13149-0090034-3

Hairi F, Ong C-HS, Suhaimi A, Tsung T-W, Ahmad MAA, Sundaraj C, Soe MM. 
2003. A knowledge, attitude and practices study on dengue among selected rural communities in Kuala Kangsar district. Pac. J. Public Health; 15(1): 37-43. DOI :

https://doi.org/10.1177\%2F10105395030 1500107

Hampson k, Coudeville L, Lembo T, Sambo M, Kieffer A, Attlan M, Barrat J, Blanton JD, Briggs DJ, Cleaveland S, Costa P, Freuling CM, Hiby E, Knopf L, Leanes F, Meslin F-X, Metlin A, Miranda ME, Müller T, Nel LH, Recuenco S, Rupprecht CE, Schumacher C, Taylor L, Vigilato MAN, Zinsstag J, Dushoff J, on behalf of the Global Alliance for Rabies Control Partners for Rabies Prevention. 2015. Estimating the Global Burden of Endemic Canine Rabies. PLoS Negl. Trop. Dis., 9: e0003786. DOI : https://doi.org/10.1371/journal.pntd.0003 786

Hampson K, Dobson A, Kaare M, Dushoff J, Magoto M, Sindoya E, Cleaveland S. 2008. Rabies exposures, post-exposure prophylaxis and deaths in a region of endemic canine rabies. PLoS Negl. Trop. Dis., 2: e339. DOI : https://doi.org/10.1371/journal.pntd.0000 339

Hergert M, le Roux K, Nel LH. 2016. Risk factors associated with nonvaccination rabies status of dogs in KwaZulu-Natal, South Africa. Vet. Med. Research and Reports, 7: 75-83. DOI : https://doi.org/10.2147/VMRR.S103859

Jibat T, Hogeveen H, Mourits MCM. 2015. Review on dog rabies vaccination coverage in Africa: A question of dog accessibility or cost recovery? PLoS Negl. Trop. Dis., 9(2) : e0003447 PLOS. DOI : https://doi.org/10.1371/journ al.pntd.0003447

Lembo T, Hampson K, Kaare MT, Ernest E, Knobel D, Kazwala RR, Haydon DT, Cleaveland S. 2010. The feasibility of canine rabies elimination in Africa: dispelling doubts with data. PLoS Negl. Trop. Dis., 4(2): e626. DOI: https://doi.org/10.1371/journal.pntd.0000 626
Mindekem R, Lechenne M, Alfaroukh IO, Moto DD, Zinsstag J, Ouedraogo LT, Salifou S. 2017. Evaluation des Connaissances-Attitudes-Pratiques des populations des districts sanitaires de Benoye, Laoukassy, Moundou et N'Djaména Sud sur la rage canine au Tchad. Pan Afr. Med. J., 27: 24. DOI: https://dx.doi.org/10.11604\%2Fpamj.201 7.27.24.11464

Minoungou G, Dahourou LD, Savadogo M, Tialla D, Comabari AHB, Kanyala E, Ouattara L, Kabore SA, Ouedraogo V, Taboure Y, Kere I. 2021. Surveillance of animal rabies in Burkina Faso: a retrospective laboratory data from 2008 to 2012. Int. J. Vet. Sci., 10(3): 172-176. DOI :

https://doi.org/10.47278/journal.ijvs/202 1.051

Mosimann L, Traoré A, Mauti S, Léchenne M, Obrist B, Véron R, Hattendorf J, Zinsstag J. 2017. A mixed methods approach to assess animal vaccination programmes: the case of rabies control in Bamako, Mali. Acta Trop., 165: 203-215. DOI: https://doi.org/10.1016/j.actatropica.201 6.10 .007

Ngwa CKF, Ogri-Okwaji M, Odugbemi T. 2010. The role of medical personnel and the environment in the transmission of healthcare-associated infections in the paediatric unit of a University Teaching Hospital. Int. J. Biol. Chem. Sci., 4(5) : 1529-1542.

DOI: https://doi.org/10.4314/ijbcs.v4i5.65535

Ojieabu WA, Eze UIH. 2011. HIV/AIDS knowledge, sexual behaviour and condom use among commercial motorcyclists in Lagos State, Nigeria. Int. J. Biol. Chem. Sci., 5(2) : 524-532. DOI: https://doi.org/10.4314/ijbcs.v5i2.72097

Rumana R, Sayeed AA, Basher A, Islam Z, Rahman MR, Faiz MA. 2013. Perceptions and treatment seeking behavior for dog bites in rural Bangladesh. Southeast Asian J. Trop. Med. Public Health, 44(2): 244248.

https://www.thaiscience.info/journals/Ar ticle/TMPH/10897750.pdf

Sabeta CT, Bingham J, Nel LH. 2003. Molecular epidemiology of canine rabies 
in Zimbabwe and South Africa. Virus Research, 91(2): 203-211. DOI : https://doi.org/10.1016/S01681702(02)00272-1

Sambo M, Lembo T, Cleaveland S, Ferguson HM, Sikana L, Simon C, Urassa H, Hampson K. 2014. Knowledge, Attitudes and Practices (KAP) about Rabies Prevention and Control: A Community Survey in Tanzania. PLoS Negl. Trop. Dis., 8 : e3310. DOI : https://doi.org/10.1371/journ al.pntd.0003310

Savadogo M, Koné P, Dahourou LD, Manishimwe R, Sow A, Nébié L, Antoine-Moussiaux N, R., BadaAlambedji R. 2020. Epidémiologie de la rage et connaissance, attitudes et pratiques des communautés au Burkina Faso. Rev. Elev. Med. Vet. Pays Trop., 73 : 133-144. DOI: http://dx.doi.org/10.19182/remvt.31863

Savadogo M, Soré A, Dahourou LD, Ossebi W, Combari AHB, Bada Alambedji R, Tarnagda Z. 2021. Assessing factors associated with owner's individual decision to vaccinate their dogs against rabies: A house-to-house survey in Ouagadougou, Burkina Faso. Veterinary World, 14(4): 1014-1019. DOI: www.doi.org/10.14202/vetworld.2021.1 014-1019

Savadogo M, Tialla D, Ouattara B, Dahourou LD, Ossebi W, Ilboudo SG, Combari AHB, Tarnagda Z, Bada-Alambedji R. 2021. Factors associated with owneddogs' vaccination against rabies: A household survey in Bobo Dioulasso, Burkina Faso. Vet. Med. Sci., 21: 1-11. DOI : https://doi.org/10.1002/vms3.468

Sondo KA, Okenga YC, Diop SA, Kaboré BE, Diallo I, Kyelem N, Basshono J, Thombiano R, Kam L. 2015. Rabies in children: report of 24 cases at the Yalgado Ouedraogo University Hospital Center of Ouagadougou in Burkina Faso. J. Trop. Dis., 3 : $168 . \quad$ DOI : http://dx.doi.org/10.4172/2329891X.1000168
Sondo KA, Zoungrana J, Bazié K, Kyelem CG, Diallo I, Korsaga N, Basshono J, Simporé L, Thiombiano R, Traoré A. 2014. Prise en charge post exposition des victimes d'agression par un animal à Ouagadougou. Science et Technique, Sciences de la Santé, 37: 27-36. https://www.ajol.info/index.php/stss/artic le/view/141168

Thys S, Sahibi H, Gabriël S, Rahali T, Lefèvre P, Rhalem A, Marcotty T, Boelaert M, Dorny P. 2019. Community perception and knowledge of cystic echinococcosis in the High Atlas Mountains, Morocco. BMC Public Health, 19: 118. DOI : https://doi.org/10.1186/s12889-0186372-y

Vigilato MA, Clavijo A, Knobl T, Silva HM, Cosivi O, Schneider MC, Leanes LF, Belotto AJ, Espinal MA. 2013. Progress towards eliminating canine rabies: policies and perspectives from Latin America and the Caribbean. Philos. Trans. R. Soc. Lond. B. Biol. Sci., 24; 368(1623): 20120143. DOI: https://doi.org/10.1098/rstb.2012.0143

Yandaï FH, Moundine K, Djoumbe E, Boulotigam K, Moukenet A, Kodindo ID, Hinzoumbe CK. 2017. Perception de risques du paludisme et utilisation des moustiquaires au Tchad. Int. J. Biol. Chem. Sci., 11(1): 228-236. DOI: https://doi.org/10.4314/ijbcs.v10i6.19

Zangré H. 2018. Enquête sur l'approche One Health et son application dans les stratégies de lutte contre la rage au Burkina Faso. Thèse Médecine Vétérinaire, EISMV, Dakar, 91p.

Zinsstag J, Dürr S, Penny MA, Mindekem R, Roth F, Menendez Gonzalez S, Naissengar S, Hattendorf J. 2011. Dynamique de transmission et coût de la lutte contre la rage chez les chiens et les hommes dans une ville africaine. Med. Trop., 71 : 596-604. 